\title{
Caractérisation physico-chimique du gypse de Tahoua
}

\author{
Ibrahim NATATOU ${ }^{1 *}$, Gourouza MAROU ${ }^{1}$, Zanguina ADAMOU ${ }^{1}$, \\ Yahaya MOUSSA ${ }^{1}$ et Anne BOOS ${ }^{2}$ \\ ${ }^{1}$ Université Abdou Moumouni, BP 10662, Niamey, Niger. \\ ${ }^{2}$ Ecole de chimie polymère et matériaux (ECPM), 25 rue Becquerel, F-67087 Strasbourg Cedex 2, France. \\ *Auteur correspondant, E-mail : inatatou@yahoo.com; Tel : (00227) 96408273.
}

\section{RESUME}

Le Gypse rencontré dans la région de Tahoua se présente sous trois formes : fibreux, nodulaire et en plaquette. Les gypses nodulaire et fibreux font l'objet de ce travail. La diffraction des rayons X (DRX) montre que l'échantillon fibreux est du gypse presque pur mais que le nodulaire contient une proportion importante d'impuretés. L'analyse chimique par ICP-AES a permis de déterminer le pourcentage de gypse $\left(\mathrm{CaSO}_{4}, 2 \mathrm{H}_{2} \mathrm{O}\right)$ dans chaque échantillon : soit $98,44 \%$ dans le fibreux et $68,40 \%$ dans le nodulaire. L'analyse par ICP-MS avec une sensibilité de l'appareil de l'ordre du ppb, a montré que les éléments chimiques suivants : $\mathrm{B}, \mathrm{Sc}, \mathrm{V}, \mathrm{Cr}$, Mn, Co, Ni, Cu, Ga, As, Se, Br, Rb, Y, Zr, Nb, Mo, Ru, Rh, Pd, Ag, Cd, In, Sb, Te, Cs, Ba, La, Ce, Pr, Nd, Sm, $\mathrm{Eu}, \mathrm{Gd}, \mathrm{Tb}, \mathrm{Dy}, \mathrm{Ho}, \mathrm{Er}, \mathrm{Tm}, \mathrm{Yb}, \mathrm{Lu}, \mathrm{Hf}, \mathrm{Ta}, \mathrm{W}, \mathrm{Re}, \mathrm{Os}, \mathrm{Ir}, \mathrm{Pt}, \mathrm{Au}, \mathrm{Hg}, \mathrm{Tl}, \mathrm{Pb}, \mathrm{Bi}$, Th et U sont à des concentrations inférieures à $1 \mu \mathrm{g} / \mathrm{Kg}$ dans chaque échantillon. Les isothermes d'adsorption et de désorption de l'azote par le gypse fibreux montrent que la surface spécifique et le volume poreux de l'échantillon sont respectivement de $21 \mathrm{~m}^{2} / \mathrm{g}$ et de $0,03 \mathrm{~cm}^{3} / \mathrm{g}$. Les analyses ATG-ATD des gypses fibreux et nodulaire révèlent une perte en masse respectivement de $20 \%$ et $17 \%$ à $138^{\circ} \mathrm{C}$.

(C) 2012 International Formulae Group. All rights reserved.

Mots clés: Gypse, analyse, minéralogie, eau.

\section{INTRODUCTION}

Le gypse entre dans la fabrication du ciment, du plâtre et d'éléments préfabriqués pour l'industrie du bâtiment (Vignes, 1997). L'addition de gypse au clinker permet en effet de réguler la prise du ciment, notamment de ceux qui contiennent des proportions importantes d'aluminate tricalcique. Dans les travaux publics, il est utilisé comme stabilisateur des routes. L'industrie chimique l'utilise pour la fabrication de l'acide sulfurique. On l'utilise également pour transformer le carbonate de potassium des sols en sulfate assimilable par les plantes. Des travaux géologiques réalisés par Faure (1965) et Diallo (1982) ont mis en évidence d'importants indices de gypse dans la vallée de la Maggia (région de Tahoua). A la suite de ces travaux, les réserves ont été estimées à plus de 46364 tonnes de gypse.

Le gypse rencontré dans les formations argilo-marneuses est de nature secondaire et se présente sous trois formes principales :

- Le gypse fibreux: En couches d'épaisseur variant de 0,5 à $8 \mathrm{~cm}$, généralement horizontales et marquant la transition entre des argilites de couleurs différentes (Delesse, 2002). 
- Le gypse en nodules: Il se présente en fragments plus ou moins globuleux de dimensions et de poids très variables; le plan de plus grande section des nodules en place est toujours sensiblement horizontal.

- Le gypse en plaquettes : Il s'agit de la forme la plus rare; les plaquettes avec le plan de plus grande section parallèle au plan horizontal, se trouvent toujours dans les argilites feuilletées, noires. L'épaisseur du banc ne dépasse guère $8 \mathrm{~cm}$ (Pias, 1972).

En effet, le gypse, les argiles, les os calcinés sont des matériaux utilisés pour la conception de pilote de défluoruration d'eau de boisson dans le cadre de l'hydraulique villageoise (Diawara et al., 2004).

L'objectif visé, dans ce travail, est la détermination de la qualité du gypse de Tahoua en vue de son utilisation dans la purification des eaux. Pour atteindre cet objectif, la détermination de certains paramètres minéralogiques, chimiques et physico-chimiques s'avère indispensable pour mieux identifier ces gypses, d'où la réalisation de cette étude de caractérisation.

\section{MATERIEL ET METHODES \\ Zones d'étude}

Dans la région de Tahoua, le gypse apparaît au cours du Maestrichtien dans les marnes de l'Ader Doutchi (bassin des Iullemmeden). Les gisements se trouvent dans une bande sub-méridienne d'environ $50 \mathrm{~km}$ de large. Depuis Madaoua au Sud, la bande passe par Bouza, Kao et se poursuit jusqu'à la frontière du Mali, en passant par Mentess (Figure 1). Cette zone correspond aux affleurements de la formation de Farin Doutchi d'âge Maestrichtien. Ses affleurements sont plus ou moins recouverts par les dépôts dunaires du Continental Terminal.

Dans la région de Bouza, la formation de Farin Doutchi a une épaisseur moyenne de $15 \mathrm{~m}$; les argilites et marnes gypsifères qui la constituent, forment en continu le flanc des collines de l'Ader Doutchi; leur épaisseur demeure relativement constante.

Des échantillons de gypse fibreux et de gypse nodulaire ont été prélevés à la station de Tazoumali dont les coordonnées GPS sont: Altitude $475 \mathrm{~m}$; Latitude N 15 ${ }^{\circ} 19^{\prime} 30,7^{\prime}$ '; Longitude E $5^{\circ} 41^{\prime}$ 21', Différentes techniques d'analyses quantitative et qualitative (DRX, IR, ATG-ATD, ICP-MS, ICP-AES, BET...) ont été utilisées, dans ce travail, pour caractériser les échantillons étudiés.

\section{Méthodes expérimentales}

L'analyse granulométrique est effectuée au moyen d'un granulomètre Laser Mastersizer 2000 de MALVERN Instruments. Les échantillons de gypse ont été broyés à l'agate puis tamisés avec un tamis de diamètre $125 \mu \mathrm{m}$. Une portion de la fraction inférieure à $125 \mu \mathrm{m}$ a été dispersée dans $100 \mathrm{ml}$ d'eau distillée puis soumise aux ultrasons pendant $30 \mathrm{mn}$. La mesure est faite à partir de la solution obtenue après dispersion par l'ultrason. La distribution granulométrique est déduite de l'interaction entre les particules et le rayonnement incident.

L'analyse par spectrométrie Infra Rouge a été effectuée sur une poudre de l'échantillon au moyen d'un spectromètre 'Universal ATR (Attenuated Total Reflectance) sampling accessory" de PERKIN ELMER.

L'analyse qualitative par fluorescence $\mathrm{X}$ à dispersion d'énergie (E.D.X.R.F) a été effectuée au moyen d'un spectromètre prototype de SIEMENS. Le tube utilisé possède une anode en molybdène.

Les diffractogrammes ont été réalisés à l'aide d'un diffractomètre RX Brüker (modèle D5000), en mode $\theta / 2 \theta$ (Balayage de 3 à $15^{\circ}$ ou $3^{\circ}$ à $30^{\circ}$, par pas de $0,02^{\circ}$ d'une durée de 1 $\mathrm{s}$, anticathode $\mathrm{Cu}, 40 \mathrm{kV}-30 \mathrm{~mA})$. L'identification a été faite à l'aide de la fiche du répertoire du Joint Comittee on Powder Diffraction Standards (JCPDS) (méthode de Hanawalt). 


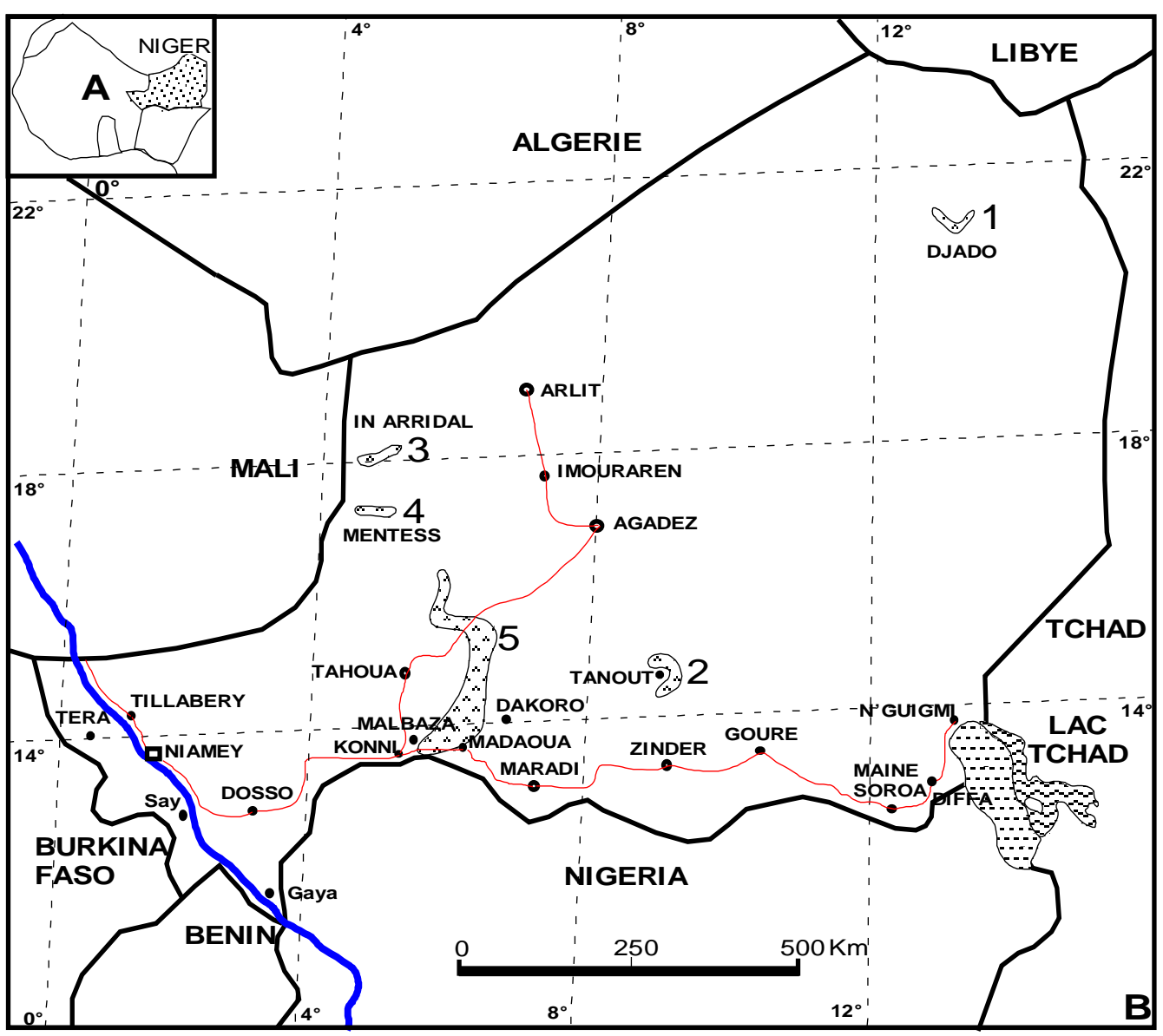

Figure 1: Localisation et répartition par ordre d'importance des principales zones gypsifères au Niger. A: Localisation du Niger en Afrique de l'Ouest; B: Répartition par ordre d'importance des principales zones gypsifères au Niger. $\mathbf{1}$ = Djado (Namuro-Westphalien); $\mathbf{2}=$ Tanout (Turonien); $\mathbf{3}=$ In Arridal (Sénonien inférieur.); 4 = Mentess (Maestrichtien); 5 = Ader Doutchi (Maestrichtien). (Carte extraite du plan minéral, modifiée).

L'analyse élémentaire a été effectuée à l'aide d'un spectromètre de masse à plasma inductif (ICP-MS) Agilent 7500 Series (analyse qualitative) et à l'aide d'un spectromètre d'émission atomique à plasma inductif (ICP-AES) Ultrace 138 de Jobin Yvon (analyse quantitative).

Les analyses thermogravimètrique (ATG) et thermodifférentielle (ATD) ont été effectuées à l'aide d'un appareil A 5c 1000c sous air. Les isothermes d'adsorptiondésorption de l'azote ont été mesurés sur un appareil Thermofinnigan, modèle Sorptomatic 1990 à $77 \mathrm{~K}$.

\section{RESULTATS ET DISCUSSION}

La granulométrie Laser consiste à déterminer la répartition granulométrique par la technique de diffraction de la lumière laser après la mise en suspension de l'échantillon dans un dispersant. La distribution granulométrique est déduite de l'interaction entre un ensemble de particules et le rayonnement incident (Dur et al., 2004).

L'analyse granulométrique par diffraction laser des deux gypses $(0,01$ à 2000 $\mu \mathrm{m})$ révèle une population principale centrée sur $100 \mu \mathrm{m}$ (Figures 2 et 3 ) caractéristique du gypse. Pour le gypse nodulaire, on observe également une population fine en quantité non 
négligeable qui apparaît dans un domaine de 4 à $12 \mu \mathrm{m}$ caractéristique des impuretés (silices ou argiles). Les résultats montrent que pour le gypse fibreux, $50 \%$ des grains ont un diamètre inférieur ou égal à $75 \mu \mathrm{m}$ et $90 \%$ ont un diamètre inférieur à $152 \mu \mathrm{m}$. L'amplitude se situe autour de $100 \mu \mathrm{m}$. Pour le gypse nodulaire, $50 \%$ des grains ont un diamètre inférieur ou égal à $83 \mu \mathrm{m}$ et $90 \%$ ont un diamètre inférieur à $223 \mu \mathrm{m}$. Comme dans le cas du gypse fibreux, la plus grande dispersion s'étend autour de $100 \mu \mathrm{m}$.

\section{Spectrométrie Infra Rouge (IR)}

Sur le spectre de l'échantillon fibreux (Figure 4) d'une part et sur celui du nodulaire (Figure 5) d'autre part, les bandes respectivement autour de : $1109,43 \mathrm{~cm}^{-1}$, $668,15 \mathrm{~cm}^{-1}, 1115,25 \mathrm{~cm}^{-1}$ et $668,99 \mathrm{~cm}^{-1}$ sont indicatrices de la présence de liaison S-O. La bande dédoublée autour de $3529,11 \mathrm{~cm}^{-1}$ et celle à $1610 \mathrm{~cm}^{-1} \mathrm{du}$ spectre de l'échantillon fibreux puis celles dédoublées autour de $3534,17 \mathrm{~cm}^{-1}$ et non dédoublées à 1620,53 $\mathrm{cm}^{-1}$ de l'échantillon nodulaire sont caractéristiques de la présence de liaison $\mathrm{O}-\mathrm{H}$ (Maglione, 1975 ; Wiegel et Kirchner, 1966).

\section{Analyse qualitative par fluorescence $X$ à dispersion d'énergie}

L'analyse qualitative par fluorescence $\mathrm{X}$ du gypse fibreux et nodulaire a permis de mettre en évidence la présence des éléments suivants selon la nature du gypse : $\mathrm{Ca}, \mathrm{S}, \mathrm{Sr}$, Fe. Il ressort de cette analyse par superposition des pics (Figure 6) que le gypse nodulaire est plus ferrugineux que le gypse fibreux. Des travaux effectués sur le phosphate naturel (Natatou et al., 2005) qui est aussi de type nodulaire montrent que ces types de matériaux ont une importante teneur en ferral ( $\mathrm{Fe}, \mathrm{Al})$ et en silice.

La quasi inexistence des métaux lourds $(\mathrm{Cd}, \mathrm{Cr}, \mathrm{Pd}, \ldots)$ est une garantie de qualité du produit pour une éventuelle utilisation de ces matériaux dans le traitement de l'eau de boisson (Sato et al., 2002; Sarvinder et al., 2004; Welch et al., 2000).

\section{Analyse par diffraction des rayons X}

Les raies $(7,61 ; 4,29 ; 3,80 ; 3,07$; $2,87 ; 2,22 ; 1,99 ; 1,81 ; 1,62) \mathrm{du}$ diffractogramme de la Figure 7 sont toutes caractéristiques du gypse. Par contre, sur la Figure 8, en plus des raies caractéristiques du gypse, on observe d'autres raies, qui présagent la présence d'impuretés.

\section{Analyse chimique}

L'analyse des échantillons par la fluorescence $X$ ayant donné une très faible gamme d'éléments chimiques, nous avons opté pour l'analyse par ICP-MS afin d'étendre cette gamme. Avec une sensibilité de l'ordre $\mathrm{du}$ ppb $(\mu \mathrm{g} / \mathrm{L})$, les résultats de l'ICP-MS montrent que les éléments suivants : $\mathrm{B}, \mathrm{Sc}, \mathrm{V}$, $\mathrm{Cr}, \mathrm{Mn}, \mathrm{Co}, \mathrm{Ni}, \mathrm{Cu}, \mathrm{Ga}, \mathrm{As}, \mathrm{Se}, \mathrm{Br}, \mathrm{Rb}, \mathrm{Y}, \mathrm{Zr}$, $\mathrm{Nb}, \mathrm{Mo}, \mathrm{Ru}, \mathrm{Rh}, \mathrm{Pd}, \mathrm{Ag}, \mathrm{Cd}, \mathrm{In}, \mathrm{Sb}, \mathrm{Te}, \mathrm{Cs}$, $\mathrm{Ba}, \mathrm{La}, \mathrm{Ce}, \mathrm{Pr}, \mathrm{Nd}, \mathrm{Sm}, \mathrm{Eu}, \mathrm{Gd}, \mathrm{Tb}, \mathrm{Dy}, \mathrm{Ho}$, Er, Tm, Yb, Lu, Hf, Ta, W, Re, Os, Ir, Pt, Au, $\mathrm{Hg}, \mathrm{Tl}, \mathrm{Pb}, \mathrm{Bi}$, Th et $\mathrm{U}$ sont pratiquement absents dans les échantillons.

L'ICP-AES est complémentaire à l'ICP-MS en vue de déterminer les proportions en certains éléments (Tableaux 1 et 2). Il ressort de ces analyses que le gypse $\left(\mathrm{CaSO}_{4}, 2 \mathrm{H}_{2} \mathrm{O}\right)$ représente $98,44 \%$ en masse de l'échantillon fibreux et $68,40 \%$ du nodulaire. Le rapport $\mathrm{Ca} / \mathrm{S}$ est de 1,31 pour le gypse fibreux et de 1,12 pour le gypse nodulaire.

\section{Analyses thermiques}

Le comportement thermique des échantillons a été étudié par analyse thermogravimétrique (ATG) et analyse thermique différentielle (ATD) (Figures 9 et 10).

Le pic endothermique à $30{ }^{\circ} \mathrm{C}$ des ATD du fibreux et du nodulaire est accompagné d'une augmentation de masse respectivement $1,56 \%$ pour le fibreux et $1,42 \%$ pour le nodulaire (courbes ATG). Cela peut s'expliquer par une oxydation de certains éléments.

Les importants pics endothermiques entre 150 et $180{ }^{\circ} \mathrm{C}$, dont le maximum se positionne à $138{ }^{\circ} \mathrm{C}$, correspondent à la désorption d'une partie des molécules d'eaux adsorbées et structurales. Le gypse 
$\left(\mathrm{CaSO}_{4} 2 \mathrm{H}_{2} \mathrm{O}\right)$ se déshydrate partiellement en formant le plâtre $\left(\mathrm{CaSO}_{4}, 1 / 2 \mathrm{H}_{2} \mathrm{O}\right)$ (Chang et al., 1999). Ce qui explique la perte de masse sur les courbes ATG. Cette perte de masse est de $20 \%$ pour le gypse fibreux et de $18 \%$ pour le gypse nodulaire.

- Les pics exothermiques vers $336{ }^{\circ} \mathrm{C}$ ne sont pas accompagnés d'une perte de masse sur les courbes ATG. Ce qui peut indiquer la présence de polymères organiques (carboxymethylcellulose), leur cristallisation ou leur interaction avec le gypse à cette température (Guggnheim et al., 2001; Yener et al., 2007; Onal et Sarikaya., 2009).

- Les pics exothermiques à $935^{\circ} \mathrm{C}$ sont aussi accompagnés d'une perte de masse : $4 \%$ pour le gypse fibreux et $2 \%$ pour le gypse nodulaire. Cela peut s'expliquer par la décomposition du gypse anhydre $\mathrm{CaSO}_{4}$ en $\mathrm{CaO}$ et $\mathrm{SO}_{3}$ (Hamad, 1981).

\section{Isothermes d'adsorption-désorption de l'azote}

L'isotherme obtenue est de type II dans la classification IUPAC (Figure 11). Il est caractéristique d'un solide possédant des pores de diamètre supérieur à $50 \mathrm{~nm}$ (Brunauer et al., 1938). La méthode BET (Barrett-EmmettTeller) (Figure 12) a été utilisée pour déterminer la surface spécifique de l'échantillon préalablement dégazé sous vide à $100{ }^{\circ} \mathrm{C}$ pendant 6 heures. La surface spécifique et le volume poreux de l'échantillon sont respectivement de $21 \mathrm{~m}^{2} / \mathrm{g}$ et de $0,03 \mathrm{~cm}^{3} / \mathrm{g}$.

La méthode t-plot (Figure 13) a été utilisée pour déterminer la surface spécifique développée par les mesopores et la surface spécifique développée par les micropores de l'échantillon. Ces valeurs sont respectivement de $5 \mathrm{~m}^{2} / \mathrm{g}$ et $0,008 \mathrm{~cm}^{3} / \mathrm{g}$. La méthode B.J.H (Figure 14) a été utilisée pour déterminer le volume cumulatif des mésopores de l'échantillon. Il est de l'ordre de $0,097 \mathrm{~cm}^{3} / \mathrm{g}$.

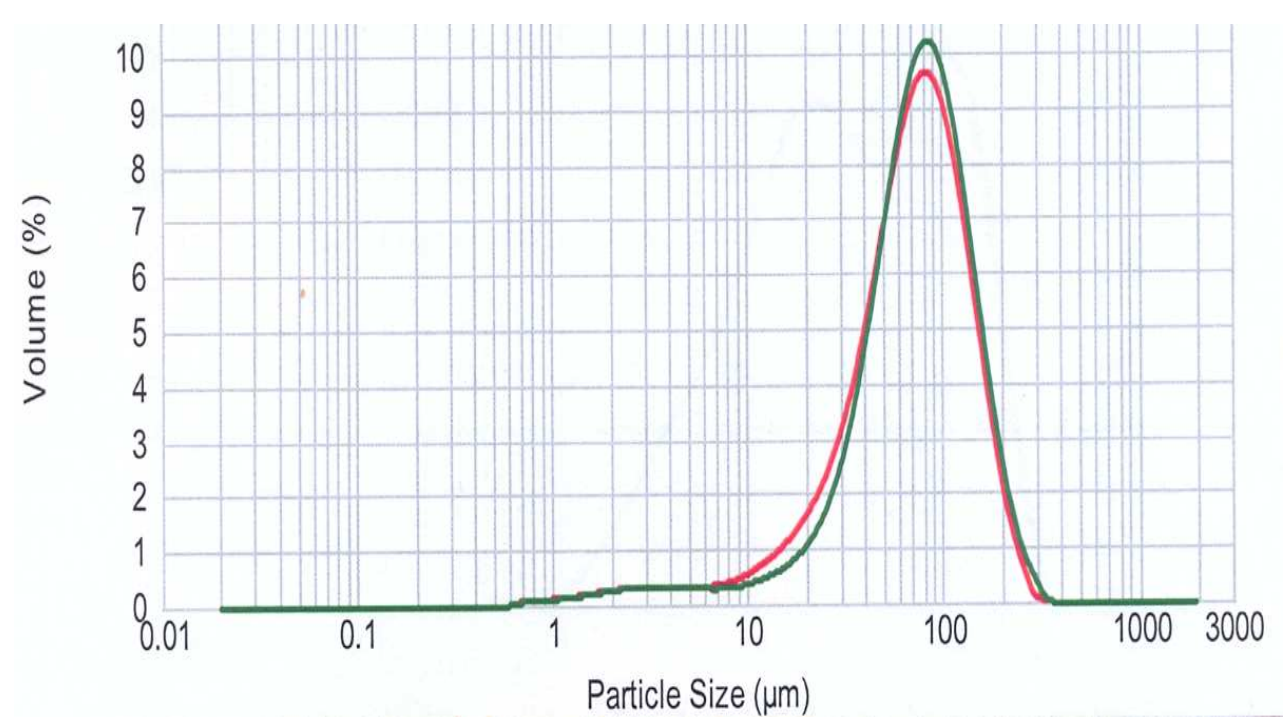

Figure 2: Granulométrie Laser du gypse fibreux. 


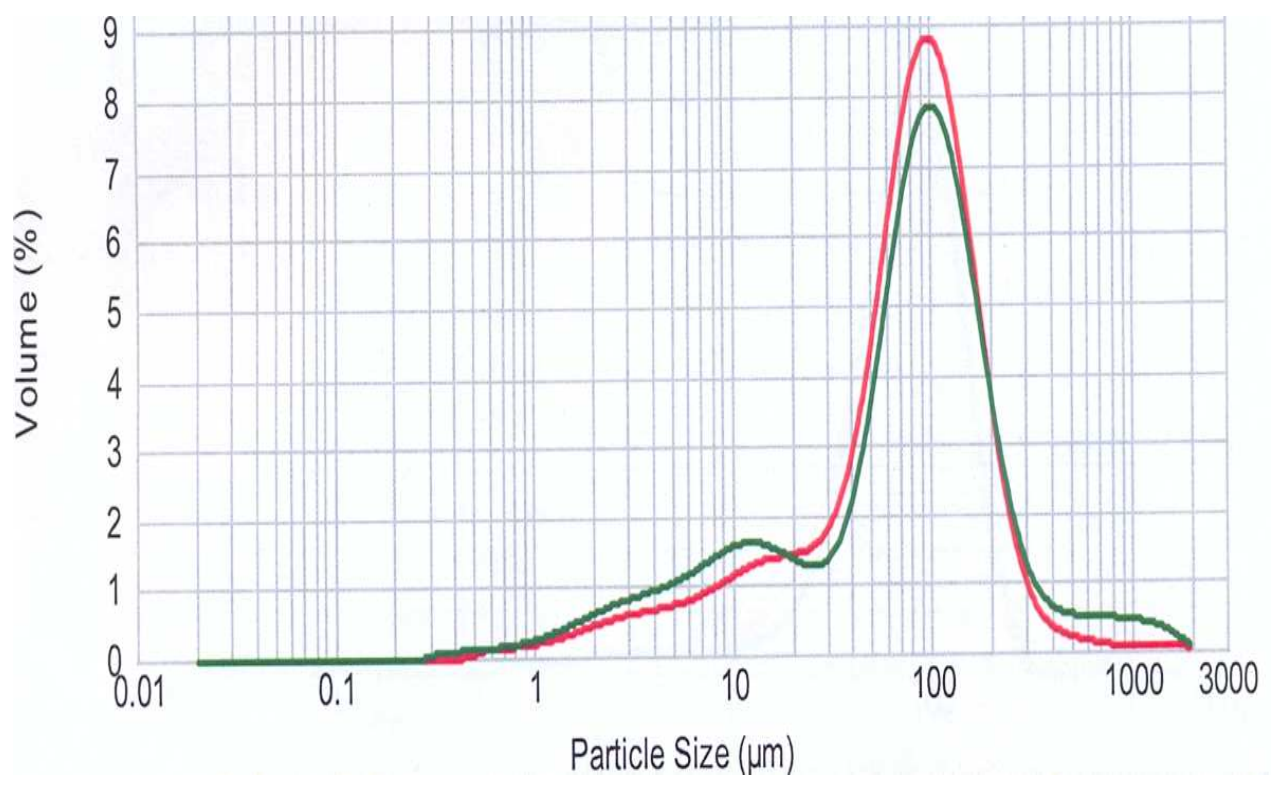

Figure 3: Granulométrie Laser du gypse nodulaire.

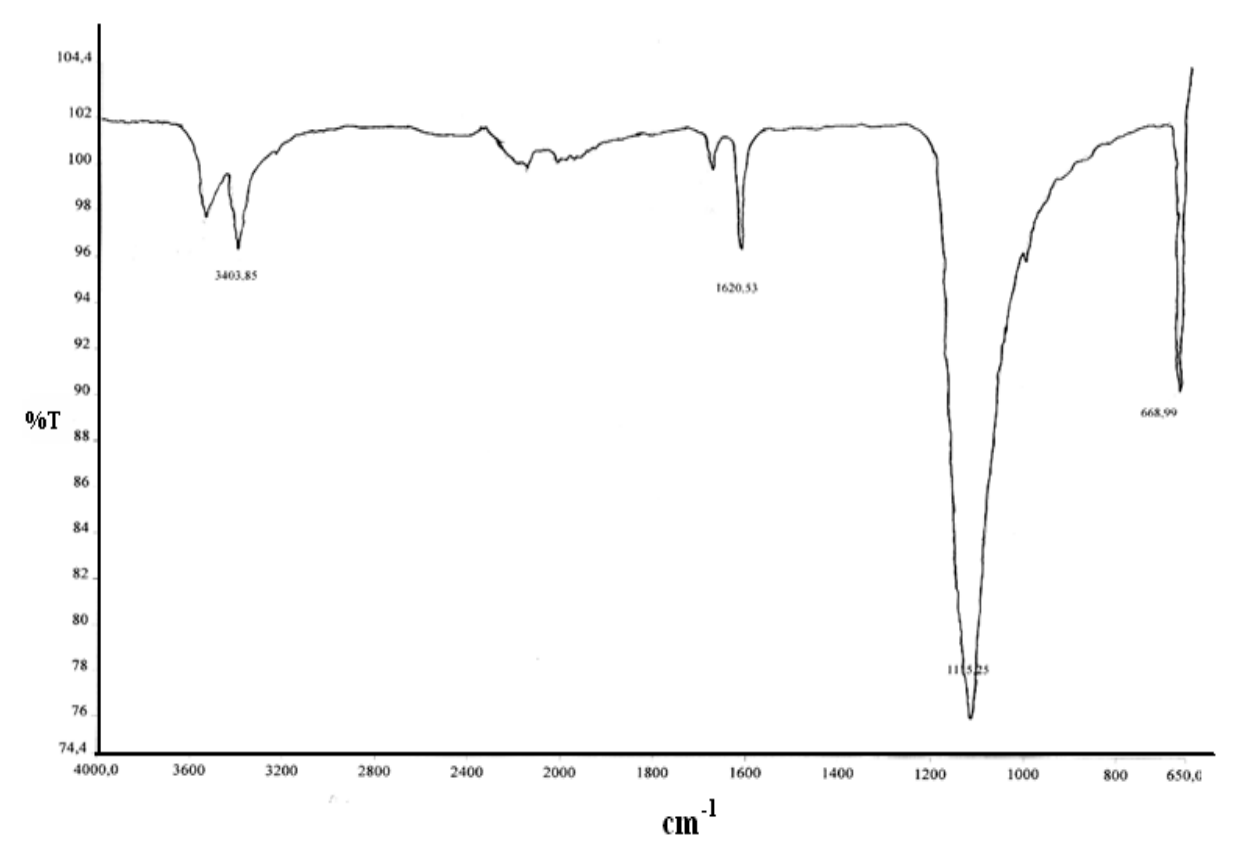

Figure 4: Spectre Infra Rouge du gypse fibreux. 


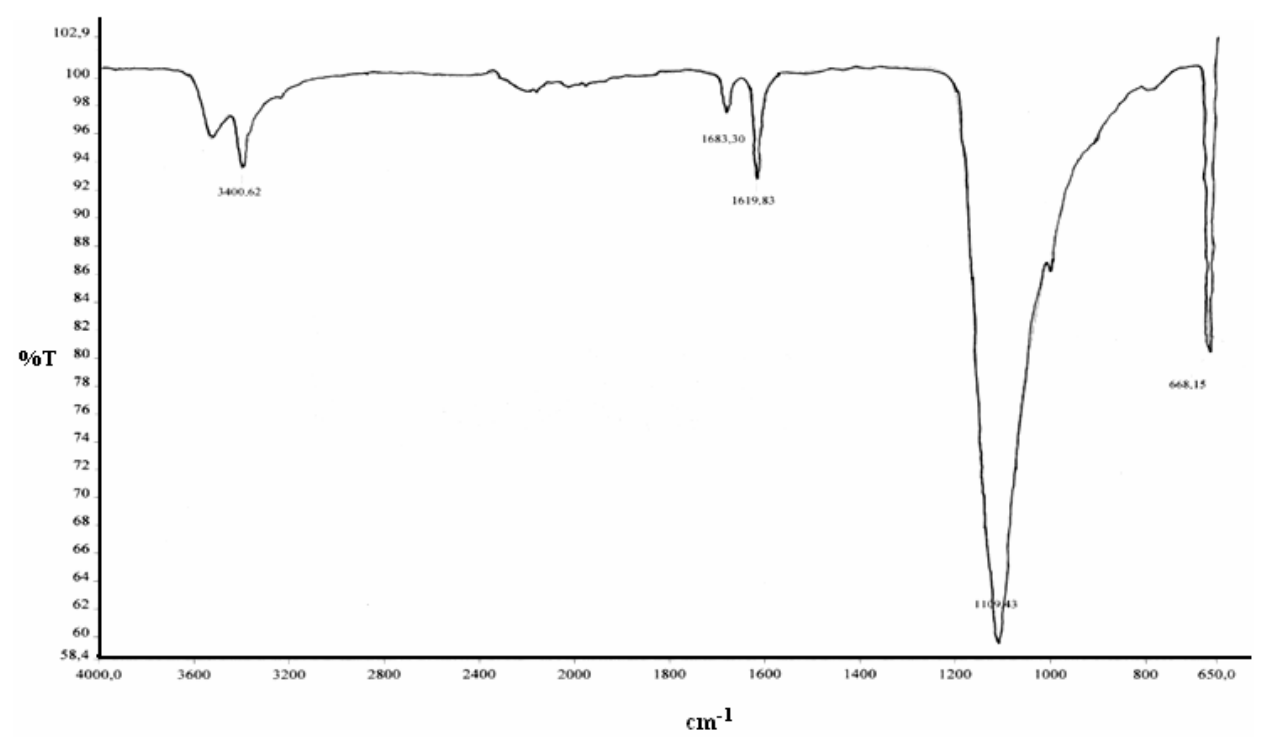

Figure 5: Spectre Infra Rouge du gypse nodulaire.

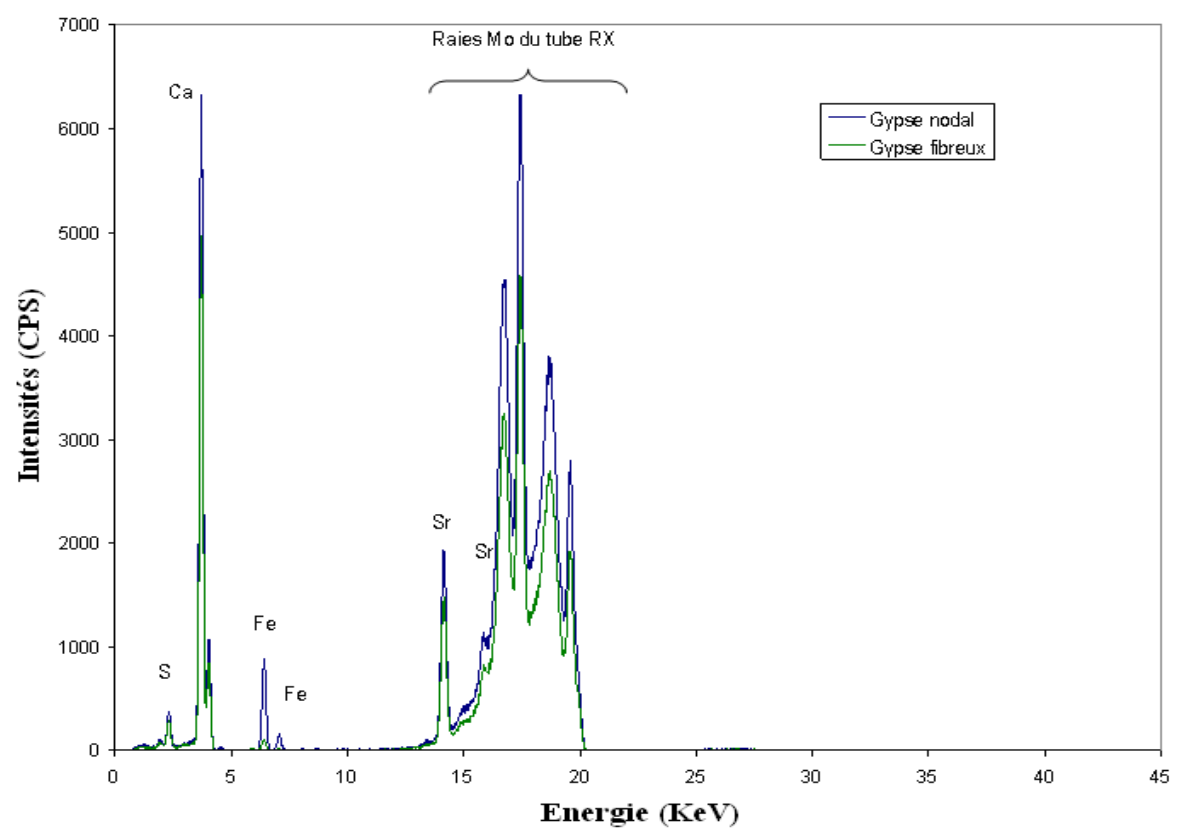

Figure 6: Analyse qualitative par fluorescence X à dispersion d'énergie (E.D.X.R.F). 


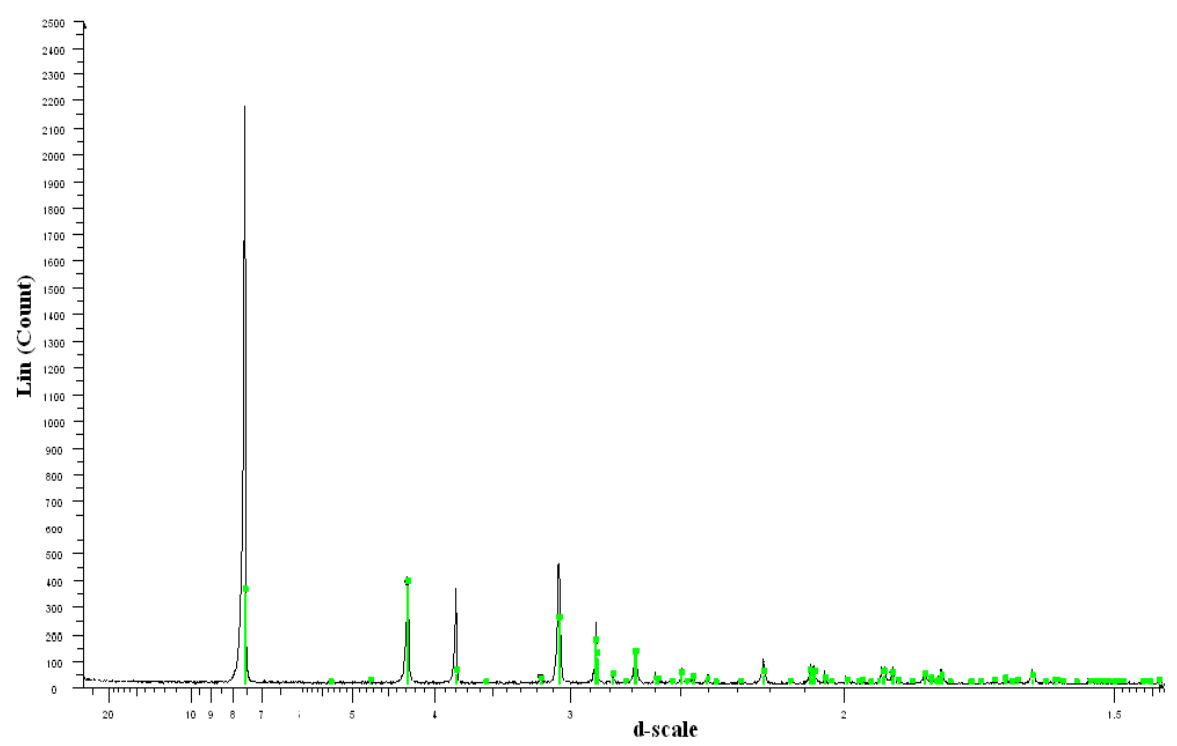

Figure 7: Diffractogramme RX du gypse fibreux.

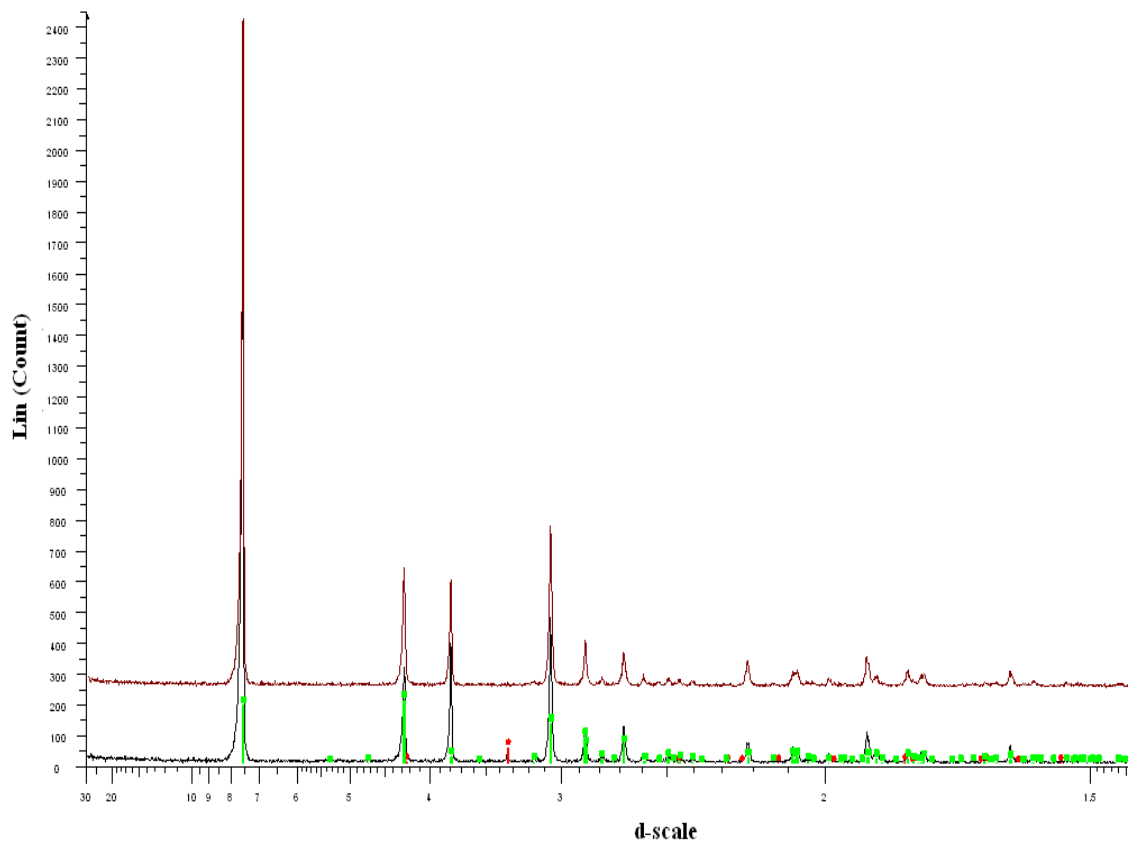

Figure 8: Diffractogramme RX du gypse nodulaire. 


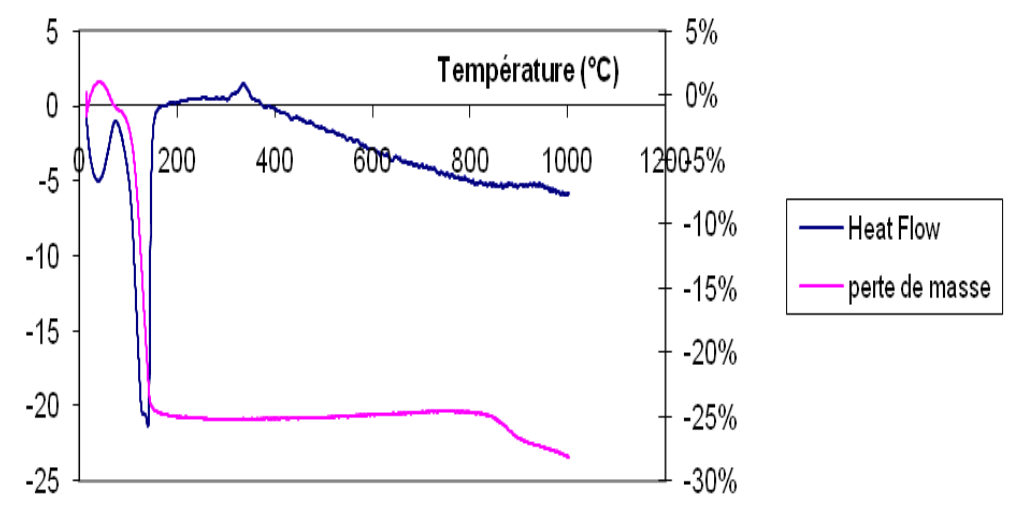

Figure 9: Thermogramme ATD - ATG du gypse fibreux.

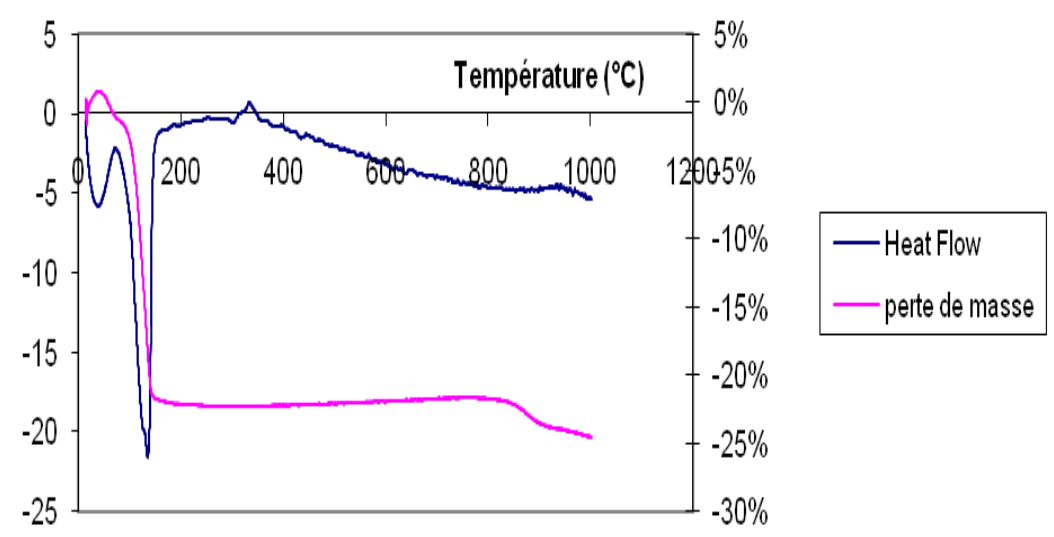

Figure 10: Thermogramme ATD - ATG du gypse nodulaire.

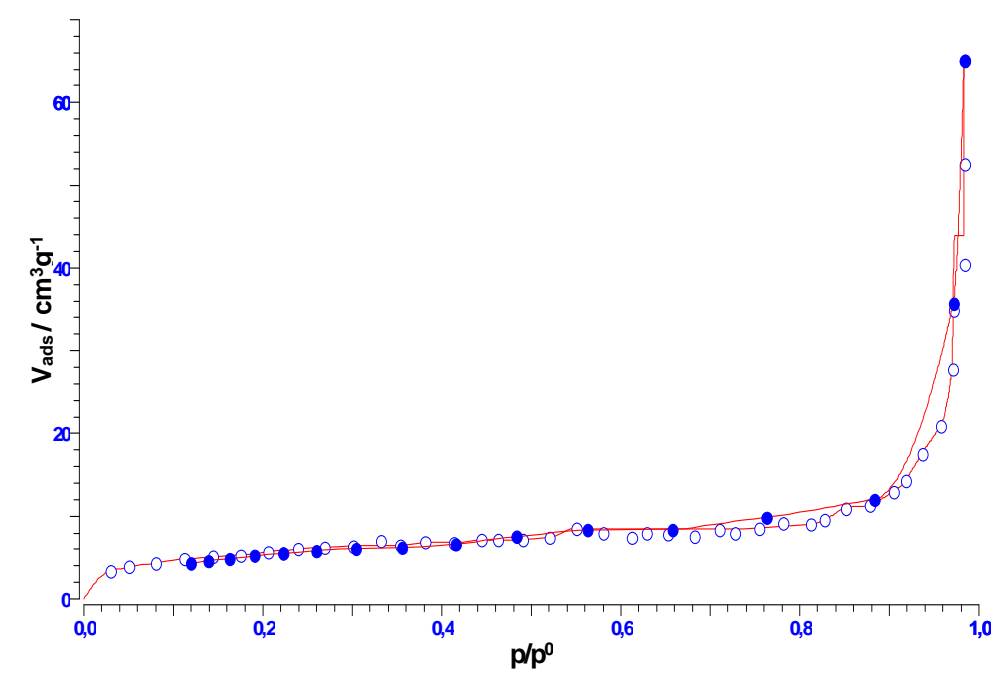

Figure 11: Isotherme d'adsorption et de désorption de la vapeur d'azote par le gypse fibreux. Les points vides matérialisent l'isotherme d'adsorption et les points pleins l'isotherme de désorption. 


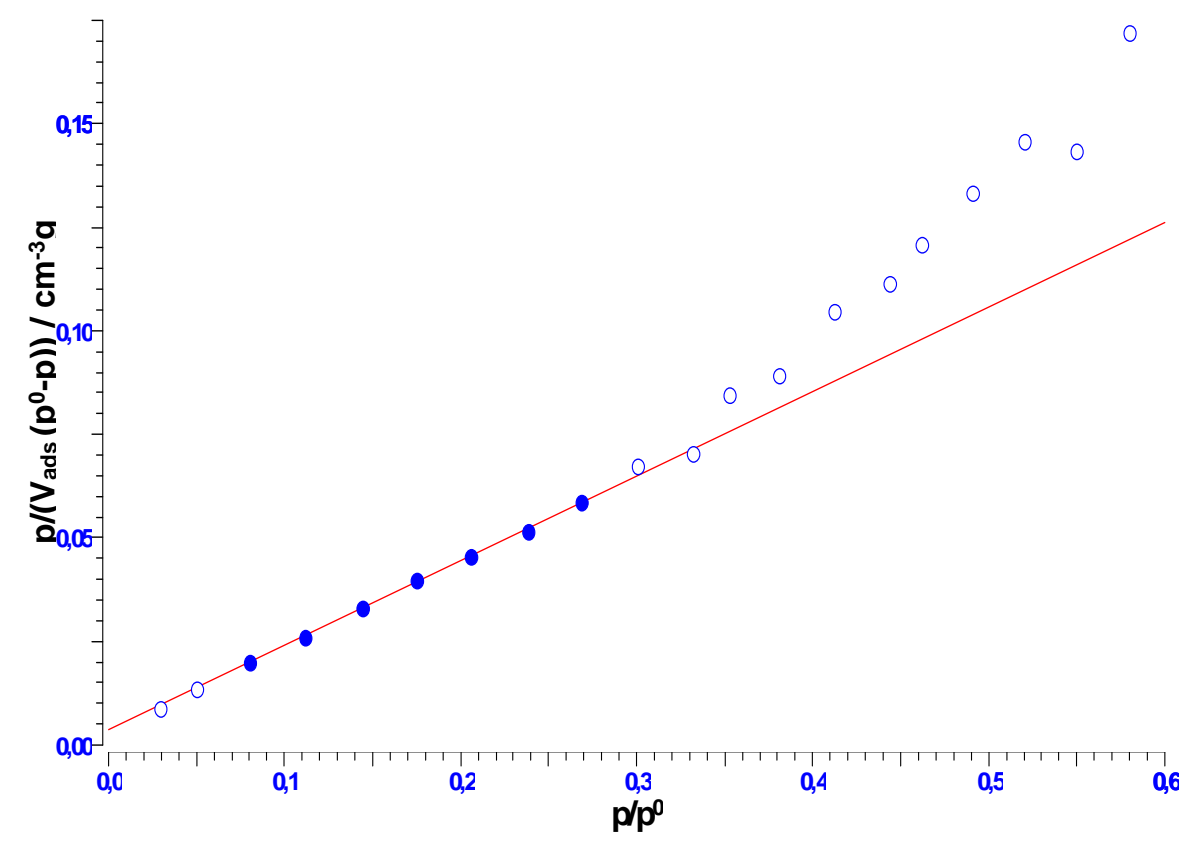

Figure 12: Application de la B.E.T au gypse fibreux.

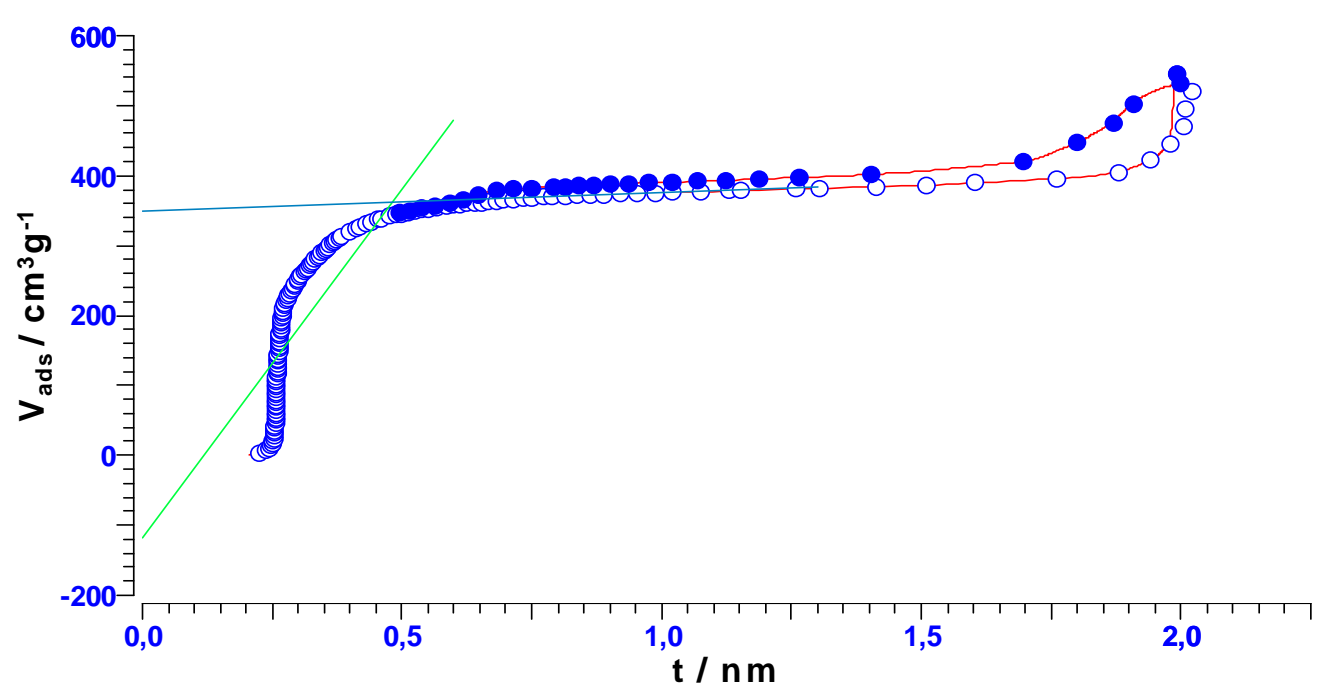

Figure 13: Application de la méthode t-plot au gypse fibreux. 


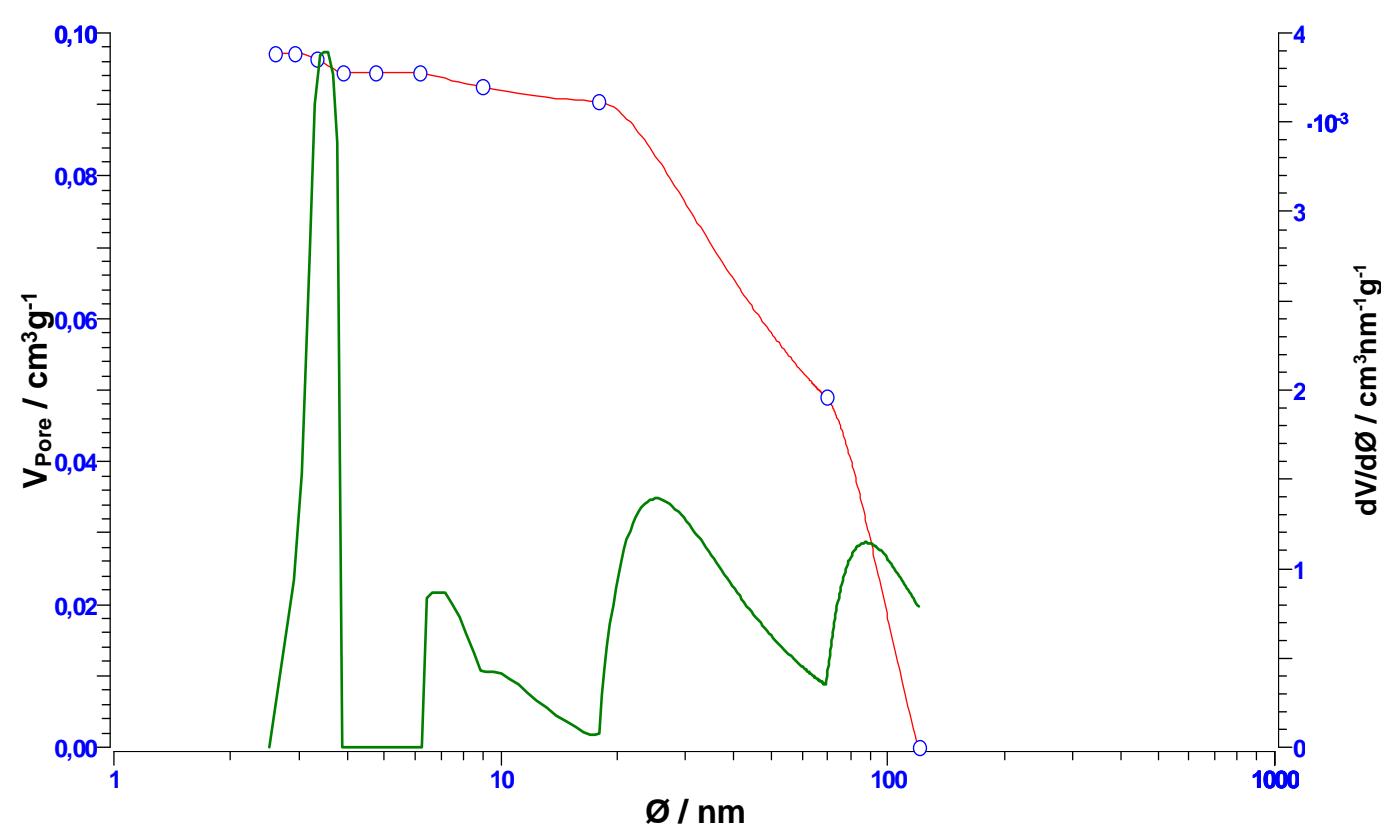

Figure 14: Application de la méthode B.J.H au gypse fibreux.

Tableau 1: Composition chimique du gypse nodulaire et fibreux par ICP-AES.

\begin{tabular}{lcc}
\hline Eléments & $\begin{array}{c}\text { Concentration }(\mathbf{m g} / \mathbf{k g}) \\
\text { Gypse nodulaire }\end{array}$ & $\begin{array}{c}\text { Concentration }(\mathbf{m g} / \mathbf{k g}) \\
\text { Gypse fibreux }\end{array}$ \\
\hline $\mathrm{Na}$ & 3,5 & 3 \\
$\mathrm{Mg}$ & 1 & 0,4 \\
$\mathrm{Al}$ & 7 & 0,66 \\
$\mathrm{Si}$ & 4,2 & 3,2 \\
$\mathrm{P}$ & 1 & 0,96 \\
$\mathrm{~S}$ & 94 & 150 \\
$\mathrm{~K}$ & 0,2 & 0,15 \\
$\mathrm{Ca}$ & 23 & 50 \\
$\mathrm{Ti}$ & 0,76 & 0,023 \\
$\mathrm{Fe}$ & 12 & 2,6 \\
$\mathrm{Zn}$ & 0,3 & 0,077 \\
$\mathrm{Sr}$ & 0,56 & 1,7 \\
$\mathrm{Sn}$ & 9,1 & 9,8 \\
\hline
\end{tabular}


Tableau 2: Composition chimique du gypse nodulaire et fibreux par ICP-MS.

\begin{tabular}{lcc}
\hline Eléments & $\begin{array}{c}\text { Concentration (mg/kg) } \\
\text { Gypse nodulaire }\end{array}$ & $\begin{array}{c}\text { Concentration } \\
(\mathbf{m g} / \mathbf{k g}) \\
\text { Gypse fibreux }\end{array}$ \\
\hline $\mathrm{Na}$ & 3,12 & 2,91 \\
$\mathrm{Mg}$ & 1,1 & 0,42 \\
$\mathrm{Al}$ & 8,06 & 0,79 \\
$\mathrm{Si}$ & 2,92 & 2,13 \\
$\mathrm{P}$ & 0,47 & 0,67 \\
$\mathrm{~S}$ & 121,03 & 174,67 \\
$\mathrm{Cl}$ & 4740,68 & 4658,02 \\
$\mathrm{~K}$ & 0,22 & 0,17 \\
$\mathrm{Ca}$ & 34,29 & 54,34 \\
$\mathrm{Sr}$ & 1,10 & 1,94 \\
$\mathrm{Sn}$ & 8,27 & 5,72 \\
$\mathrm{I}$ & 0,53 & 0.54 \\
$\mathrm{C}$ & 0,36 & 0,37 \\
$\mathrm{Fe}$ & 13,11 & 3,00 \\
$\mathrm{Zn}$ & 0,33 & 0,08 \\
\hline
\end{tabular}

\section{Conclusion}

Les différentes techniques d'analyse (DRX, IR, ATG-ATD, ICP-MS, ICP-AES, BET...) utilisées dans ce travail de caractérisation permettent de conclure que des deux types de gypse de la région de Tahoua, le fibreux est du gypse presque pur avec $98,44 \%$ de $\mathrm{CaSO}_{4}, 2 \mathrm{H}_{2} \mathrm{O}$. Il est par conséquent de qualité supérieure par rapport au nodulaire présentant $68,40 \%$ de $\mathrm{CaSO}_{4}, 2 \mathrm{H}_{2} \mathrm{O}$. Les principales impuretés du nodulaire sont $\mathrm{du}$ ferral ( $\mathrm{Fe}, \mathrm{Al})$.

Les résultats des analyses chimiques permettent l'utilisation de ces produits pour la conception de pilote de purification d'eau de boisson dans le cadre de l'hydraulique villageoise.

\section{REFERENCES}

Brunauer S, Emmett PH, Teller E. 1938. Adsorption of gases in multimolecular layers. Journal of the American Chemical Society, 60: 308-319.

Chang H, Huang PJ, Hou SC. 1999. Application of thermo-Raman spectroscopy to study dehydration of
CaSO4_2H2O and CaSO4_0.5H2O. Materials Chemistry and Physics, 58: 1219.

Delesse A. 2002. Etudes sur le métamorphisme des roches (partie III). http://herve.delboy.perso.sfr.fr/delesse_1. html

Diallo M. 1982. Mission 301, Ader. Rapport de fin de Campagne 1981-1982. ONAREM, Niamey.

Diawara CK, Essi-Tome H, Lhassani A, Pontie M, Buisson H. 2004. Filtrations membranaires et qualité de l'eau de boisson en Afrique : Cas du fluor au Sénégal. MSAS'2004.

Dur JC, Elsass F, Chaplain V, Tessier D. 2004. The relationship between particle size distribution by lazer granulometry and image analysis by transmission electron microscopy in a soil clay fraction. European Journal of Soil Science, 55: 265-270.

Faure H. 1965. Inventaire des évaporites du Niger. D.R.G.M., A7: 25-32.

Guggnheim S, Van GROSS ASK. 2001. Baseline studies of the clay mineral 
society source clays: Thermal analysis. Calys and Clay Minerals, 49: 433-443.

Hamad SED. 1981. A study of solid phases in the system, CaSO4-H2O: I. Dehydration and hydration behavior of the hemihydrate and soluble anhydrite. Trans. J. Br. Ceram. Soc., 80: 51-55.

Maglione G, Carn M. 1975. Spectres infrarouges des minéraux salin et des silicates néoformés dans le bassin Tchadien. Cah. ORSTOM, Sér. Géol., 7(1) : 3-9.

Natatou I, Adamou Z, Ikhiri K, Boos A, Guille J, Burgard M. 2005. Caractérisation du phosphate naturel de Tahoua (Niger). Ann. Chim. Sci. Mat., 30(1): 67-76.

Önal M, Sarikaya Y. 2009. Some physicochemical properties of a clay containing smectite and palygorskite. Applied Clay Science, 44: 161-165.

Pias J. 1972. Sols d'Afghanistan pédogenèses anciennes et actuelles. Revue de Géographie Physique et de Géologie Dynamique, 14(4) : 433-1.42.

Sarvinder T, Singh TS, Pant KK. 2004. Equilibrium, kinetics and thermodynamic studies for adsorption of As III on activated alumina. Separation and Purification Technology, 36(2): 139-147.

Sato Y, Kang M, Kamei T, Magara Y. 2002. Performance of nanofiltration for arsenic removal. Water Research, 36: 3371-3377.

Vignes JL. 1997. Une vie de plâtre: expériences de chimie sur l'élaboration et la mise en œuvre d'un matériau. Bulletin de l'Union des Physiciens, 91: 145-164.

Welch AH, Westjohn DB, Helsel DR, Wanty RB. 2000. Arsenic in ground water of the United States-occurrence and geochemistry. Ground Water, 38(4): 589604.

Wiegel E, Kirchner HH. 1966. Infrared spectroscopy in the study of the thermal dehydration of gypsum. Ber. Dtsch. Keram. Ges., 43: 718-723.

Yener N, Önal M, Üstunisik G, Sarikaya Y. 2007. Thermal bihavior of mineral mixture of sepiolite and dolomite. Journal of Thermal Analysis and Calorimetry, 83: 813-817. 